\title{
Comparison of Computational Results with a Low-g, Nitrogen Slosh and Boiling Experiment
}

\author{
Mark. E. M. Stewart ${ }^{1}$ \\ VPL at NASA Glenn Research Center, Cleveland, Ohio, 44135 \\ Jeffrey P. Moder ${ }^{2}$ \\ NASA Glenn Research Center, Cleveland, Ohio, 44135
}

\begin{abstract}
This paper compares a fluid/thermal simulation, in Fluent, with a low-g, nitrogen slosh and boiling experiment. In 2010, the French Space Agency, CNES, performed cryogenic nitrogen experiments in a low-g aircraft campaign. From one parabolic flight, a low-g interval was simulated that focuses on low-g motion of nitrogen liquid and vapor with significant condensation, evaporation, and boiling. The computational results are compared with high-speed video, pressure data, heat transfer, and temperature data from sensors on the axis of the cylindrically shaped tank. These experimental and computational results compare favorably. The initial temperature stratification is in good agreement, and the two-phase fluid motion is qualitatively captured. Temperature data is matched except that the temperature sensors are unable to capture fast temperature transients when the sensors move from wet to dry (liquid to vapor) operation. Pressure evolution is approximately captured, but condensation and evaporation rate modeling and prediction need further theoretical analysis.
\end{abstract}

\section{Nomenclature}

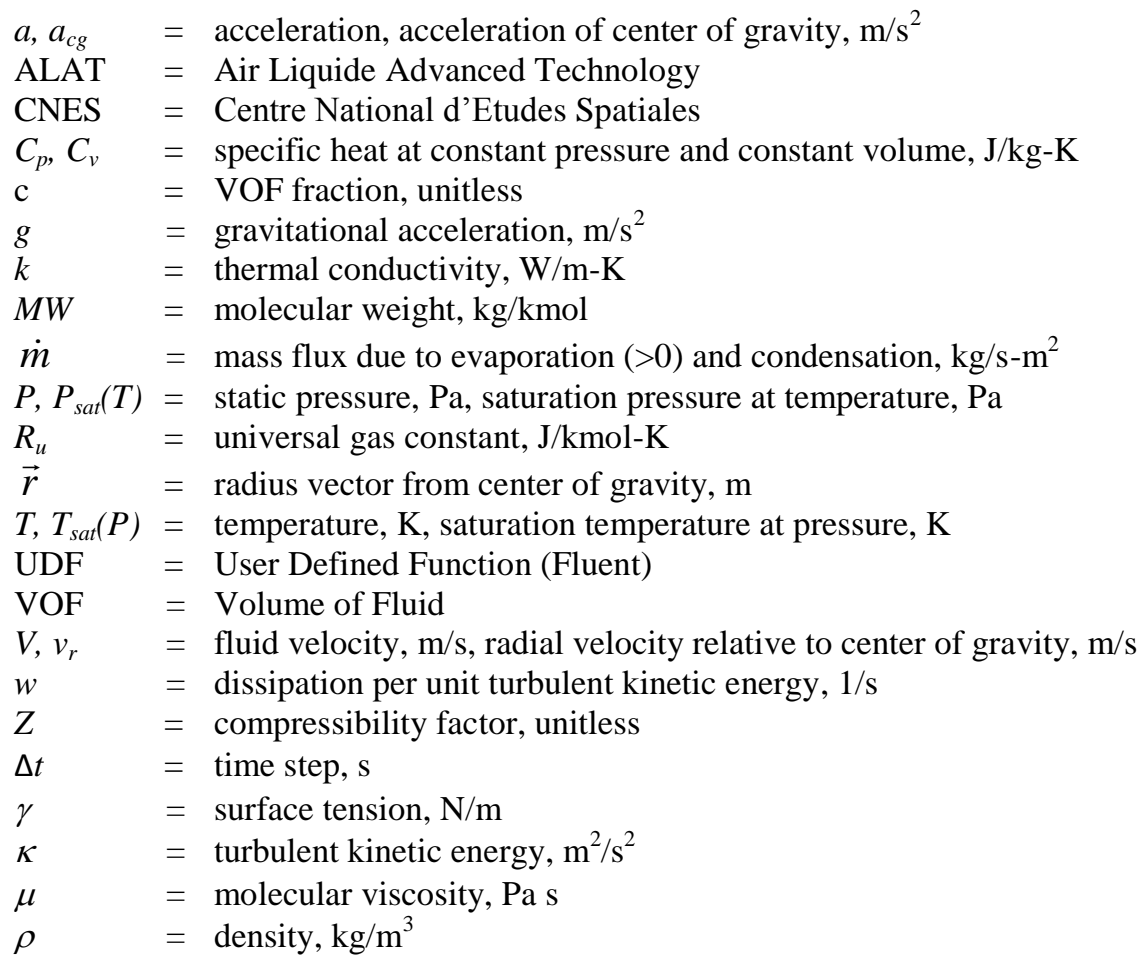

\footnotetext{
${ }^{1}$ Senior Research Engineer, MS VPL-3, AIAA member.

${ }^{2}$ Research Aerospace Engineer, Engine Combustion Branch, MS 5-10, AIAA Member.
} 


$$
\begin{aligned}
& \sigma_{\text {cond },} \sigma_{\text {evap }}=\text { accommodation coefficients for condensation and evaporation, unitless } \\
& \begin{aligned}
\tau & =\text { time constant for temperature sensor, } \mathrm{s} \\
\vec{\omega}, \dot{\vec{\omega}} & =\text { angular velocity vector, } \mathrm{rad} / \mathrm{s}, \text { angular acceleration vector, } \mathrm{rad} / \mathrm{s}^{2} \\
\text { liq, vap } & =\text { subscripts indicating liquid and vapor phases }
\end{aligned}
\end{aligned}
$$

\section{Introduction}

TN 2010, Centre National d'Etudes Spatiales and Air Liquide Advanced Technologies conducted a parabolic flight 1 campaign which included an experimental test bench, Cry0genic, to study nitrogen sloshing and boiling in low-g conditions. Computational fluid dynamics simulations accompanied these experiments [1] [2]. This experimental and computational work is part of a long term effort by CNES to examine cryogenic fluid behavior in low-g conditions, including evaporation, condensation, boiling, stratification, pressurization and de-pressurization, as well as helium pressurization.

CNES is interested [3] in cryogenic fluid behavior in flight conditions, gathering data for validating computational simulations, and predicting tank pressure, thermal stratification, and tank outlet/engine inlet conditions for engine restart after an orbital coast phase. NASA is interest in long term storage of cryogenic propellants for on-orbit refueling and long duration space missions. Cryogenic propellants promise higher specific impulse than storable hypergolic fuels, but storage for long duration missions must be demonstrated. A pacing technology for manned Mars missions using Nuclear Thermal Propulsion is storage of many tons of liquid hydrogen propellant for several years with minimal boil-off loss. With this convergence of interests, NASA and CNES agreed to benchmark simulations, and this is one of the test cases.

\section{Geometry and Grid}

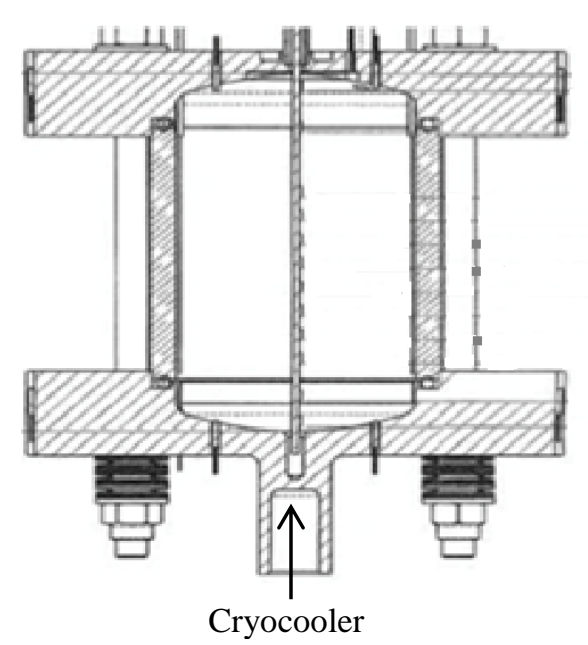

Figure 1. Cross-sectional geometry for experimental apparatus. Picture from Mathey et al. [1].
The experimental apparatus consisted of nitrogen, gas and liquid, in a sapphire cylindrical shell with aluminum and stainless steel (inox) lids (Figure 1) all contained in an insulating vacuum chamber. To remove the small residual heat fluxes into the vessel (and reduce boil off), the lower lid contains a cryocooler. Instrumentation included high speed video and twelve temperature sensors mounted to a post along the axis of the shell. The dimensions of the cylindrical nitrogen vessel are approximately $6 \mathrm{~cm}$ by $10 \mathrm{~cm}$, with a slosh frequency of near $4 \mathrm{~Hz}$.

The three-dimensional grid is shown in Figure 2. It is a full 360degree sector of the apparatus with 569,110 fluid cells, and 685,858 solid cells. The solid grid is unstructured with variable resolution. The interior of the fluid grid is a uniform, structured grid with $\sim 1 \mathrm{~mm}$ resolution, but near the fluid-solid interface, it becomes unstructured and merges into the solid grid. The grid does not include the central post where the fluid temperature sensors are mounted. The region of the joints and sealing gaskets between the sapphire shell and metal lids is meshed; however, thermal isolation is enforced along the interface lines shown in Figure 2. The grid was partitioned to run of 16 or 32 processors.

\section{Numerical Methods and Fluent Settings}

ANSYS Fluent version 13 [4] was used to solve thermal equations in the solid coupled to thermal/fluid equations in the fluid region. While the energy equation is solved in the solid region, mass, momentum, energy, and turbulence equations are solved in the fluid region with second-order upwind schemes. The PISO scheme was used for the pressure-velocity coupling, and the PRESTO! scheme was used for the pressure interpolation. The gas phase was modeled as an ideal gas, and a Boussinesq approximation was used for the liquid phase. Two-phase flow was resolved using the Volume of Fluid method [5]. The $\kappa-w$ SST turbulence model of Menter [6] [7] was used with a turbulent damping coefficient of 10. The simulation was time-accurate (second order implicit temporal scheme) with a time step $\Delta t=1.0 \times 10^{-4}$ seconds. The simulation was run on Pleiades at the NASA Advanced Supercomputing Facility. Add execution time. 


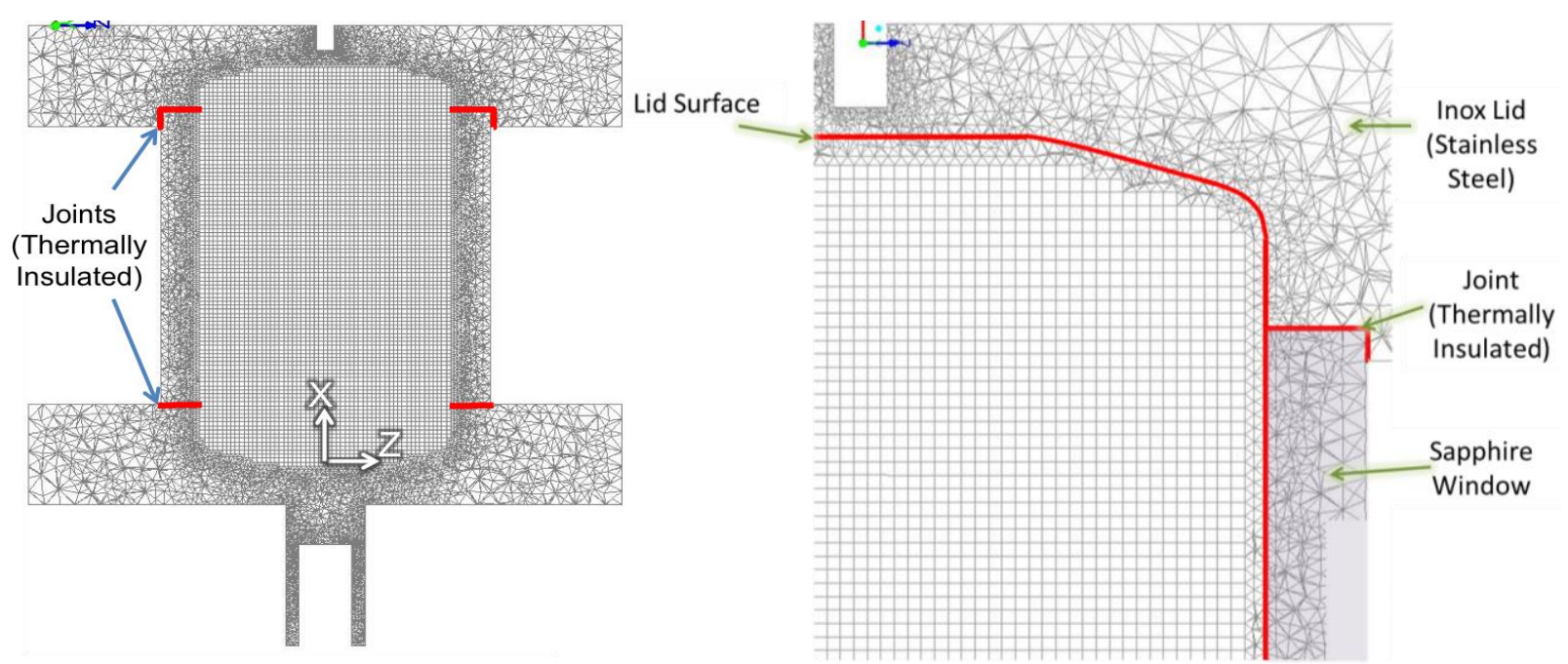

Figure 2. Grid cross-section for fluid and solid regions of experimental apparatus. Grid is 3-dimensional and a full 360 degree sector.

The following sections explain the fluid and material properties used, mass and heat transfer due to evaporation and condensation, aircraft low-g acceleration, and thermal, boundary, and initial conditions.

\section{A. Temperature and Pressure Dependence of Thermophysical Properties}

Constant fluid physical properties at reference conditions $\left(77.224 \mathrm{~K}, 10^{5} \mathrm{~Pa}\right)$ are in error [8] by as much as $10 \%$ at extreme simulation conditions $\left(110 \mathrm{~K}, 3 \times 10^{5} \mathrm{~Pa}\right)$. However, linear temperature variations accurately represent viscosity, $\mu$, thermal conductivity, $k$, surface tension, $\gamma$, and the liquid's specific heat, $C_{p}$. There are both temperature and pressure variations for the gas's specific heat, $C_{p}$, the heats of vaporization and condensation. The compressibility factor, $Z$, varies about $8 \%$ over the temperatures and pressures in the experiment. $Z$ was not corrected (ideal gas assumption), but temperature variations were included for the gas's $C_{p}$ and the heats of vaporization and condensation.

For the solid materials, temperature dependent data (specific heat, $C_{p}$, and thermal conductivity, $k$ ) were provided by CNES [9], and 310 stainless steel agrees with this data. But NIST [10] and MMPDS [11] data do not agree with the aluminum thermal conductivity.

The saturation line conditions for nitrogen, $P_{\text {sat }}(T)$ and $T_{\text {sat }}(P)$, were fit to Reynolds [12] and NIST [8] data, respectively. This is a significant improvement over the Clausius-Clapeyron equation, particularly away from reference conditions.

\section{B. Mass Transfer and Heat of Vaporization / Condensation}

The rate of mass transfer and heats of vaporization/condensation are calculated from the Schrage equation, Eq. (1), which is derived, in turn, from Maxwell's distribution. This evaporation/condensation internal boundary condition enforces saturation conditions at the liquid/vapor interface, and it is implemented in a Fluent UserDefined-Function (UDF).

Equation (1) is modified by assuming $\sigma_{\text {cond }}=\sigma_{\text {evap }} ; T_{\text {vap }}=T_{\text {liq }} ; P_{\text {vap }}=P_{\text {sat }}$. Implementation requires a length scale, $\operatorname{sqrt}\left(1 /|\operatorname{grad} c|^{2}\right)$, where $c$ is VOF fraction, to convert the mass flux $\left(\mathrm{kg} / \mathrm{s}-\mathrm{m}^{2}\right)$ to a volumetric rate, that is, to distribute the mass flux over the width of the liquid/vapor interface [13].

$$
\dot{m}_{\text {net }}=\frac{2}{2-\sigma_{\text {cond }}} \sqrt{\frac{M W_{\text {vap }}}{2 \pi R_{u}}}\left(\sigma_{\text {cond }} \frac{P_{\text {vap }}}{\sqrt{T_{\text {vap }}}}-\sigma_{\text {evap }} \frac{P_{\text {liq }}}{\sqrt{T_{\text {liq }}}}\right)
$$

Unfortunately, Eq. (1) and its implementation are not predictive of mass transfer due to evaporation or condensation across an interface in this simulation, and results are matched by adjusting the accommodation coefficient, $\sigma$. The best-fit values of the accommodation coefficient are $\sigma=1.0 \times 10^{-4}$ for evaporation and condensation at the liquid/vapor interface and for vapor phase condensation (small in this simulation). For boiling (evaporation initiated away from the liquid/vapor interface), which dominates in this simulation, $\sigma=5.0 \times 10^{-3}$ was 
used. This boiling model includes a superheat criterion, $T_{\max }-T_{\text {sat }}(P)>5 \mathrm{~K}$, that is, the liquid temperature within the cell must exceed the saturation temperature by a fixed temperature in the liquid phase. $T_{\max }$ is the maximum temperature in the cell, including adjacent walls. The vapor phase condensation model includes a similar supercooled liquid criteria based on the minimum temperature.

Data exists for boiling heat transfer rates for 1-g, nitrogen boiling in wires [14] and cylinders [15]; however, this wire and cylinder data is not clearly applicable here, so they can only provide guidance.

\section{Non-Inertial Reference Frame Accounts for Low-g Aircraft Acceleration}

A non-inertial reference frame will account for the acceleration of the aircraft during low- $g$ (and high- $g$ ) parabolic flight. In the general case, such as a satellite with measured linear acceleration, $\vec{a}_{c g}$, angular velocity, $\vec{\omega}$, and angular acceleration, $\dot{\vec{\omega}}$, the acceleration components are given by Eq. (2). The terms included on the RHS of the $\mathrm{x}-, \mathrm{y}$-, and $\mathrm{z}$-momentum equations are $\rho \vec{a}$, and in the energy equation, $\rho \vec{a} \cdot \vec{V}$.

In this experiment, angular velocity and acceleration are zero. Only two components of the linear acceleration of the aircraft, $a_{x}$ and $a_{z}$, were measured, and $a_{y}$ is assumed to be zero. The acceleration components were measured during parabolic flight at a $10 \mathrm{~Hz}$ sampling rate, as shown in Figure 3. Values are interpolated as piece-wise linear fits. The steady initial

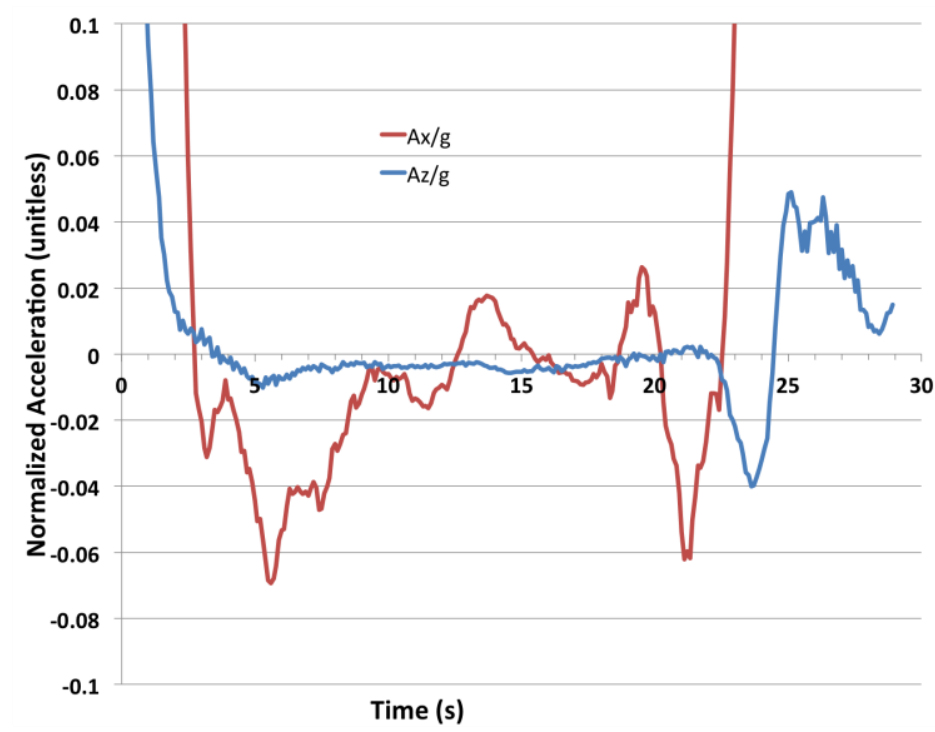

Figure 3. Acceleration profile during low-g interval. High-g refers to the preceding and following intervals. conditions assumed in the high- $g$ phase of parabolic flight are $a_{x}=-16.5 \mathrm{~m} / \mathrm{s}^{2}$ and $a_{z}=-1.93 \mathrm{~m} / \mathrm{s}^{2}$.

$$
\vec{a}=\vec{a}_{c g}+2 \vec{\omega} \times \vec{v}_{r}+\dot{\vec{\omega}} \times \vec{r}+\vec{\omega} \times(\vec{\omega} \times \vec{r})
$$

The Bond or Eötvös number is in the range $[0.3,6$. during the low- $g$ phase, and it is approximately 1 for about 10 seconds.

The weightless interval of parabolic flight is referred to as low- $g$, while high- $g$ refers to the intervening interval.

\section{Thermal, Boundary, and Initial Conditions}

Despite being contained in a vacuum chamber, small heat fluxes exist into the experimental apparatus, and they are compensated by a cryocooler attached to the lower aluminum lid. The surface heat fluxes are due to radiation from the vacuum chamber inner surface, and conduction through the near vacuum conditions. Consequently, heat flows through the apparatus as discussed in the next section. The cryocooler removes about $4 \mathrm{~W}$, and the surface heat inflow is estimated and assumed to be uniformly distributed over the top lid (1 $\mathrm{W})$, bottom lid $(1 \mathrm{~W})$, and the sapphire surface $(2 \mathrm{~W})$.

Nitrogen fills the tank $55 \%$ full. The liquid-tovapor contact angle is taken as 5 degrees. Between low$g$ intervals in the parabolic flight, the conditions are

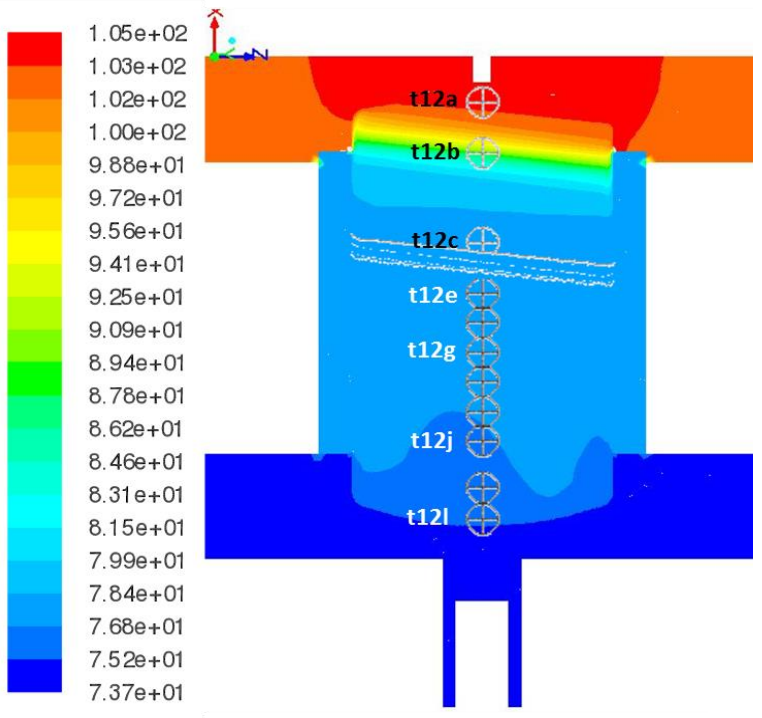

Figure 4. Initial temperature determined from a transient simulation of the high-g interval. Temperature sensor locations are also marked. Liquid/vapor interface indicated between sensors $t 12 c$ and t12e. High temperature gradient near $112 b$. 
assumed to be steady, yet since $a_{z} \neq 0$, the liquid/vapor interface is tilted. This interface position and the initial thermal conditions are calculated with a transient analysis, and a steady thermal/fluid solution was achieved well within the 90 seconds of physical time simulated. This initial temperature distribution is shown in Figure 4 . This initial solution was extended into the transient, low- $g$ simulation, that is, the non-inertial reference frame UDF commenced with non-constant values of $a_{x}$ and $a_{z}$.

\section{Operational Behavior of the Experimental Apparatus}

\section{E. How Does Boiling Occur in Low-g Conditions?}

Thermal isolation of the sapphire shell from the metal lids requires a large temperature difference for heat to diffuse through the relatively non-conductive nitrogen gas during the high- $g$ interval. Consequently, a hot, top lid develops during the high- $g$ interval (Figure 4), and boiling occurs during low- $g$ conditions when liquid nitrogen reorients and impinges on this hot lid.

A thermal analysis of the apparatus confirms this scenario for boiling in low- $g$ conditions. Small heat fluxes enter the external surfaces of the sapphire shell and lids, and this heat is removed by the cryocooler. What path will this heat take through the solid walls or the nitrogen gas and liquid? The thermal conductivity of the sapphire shell and aluminum and stainless steel lids is at least 3 orders of magnitude greater than the nitrogen vapor's conductivity (Figure 5-left), and two orders of magnitude greater than nitrogen liquid [9] [8] [12]. A spreadsheet analysis shows that if either lid thermally contacts the sapphire shell, the heat should quickly diffuse through the solid walls with a temperature difference of less than a degree.
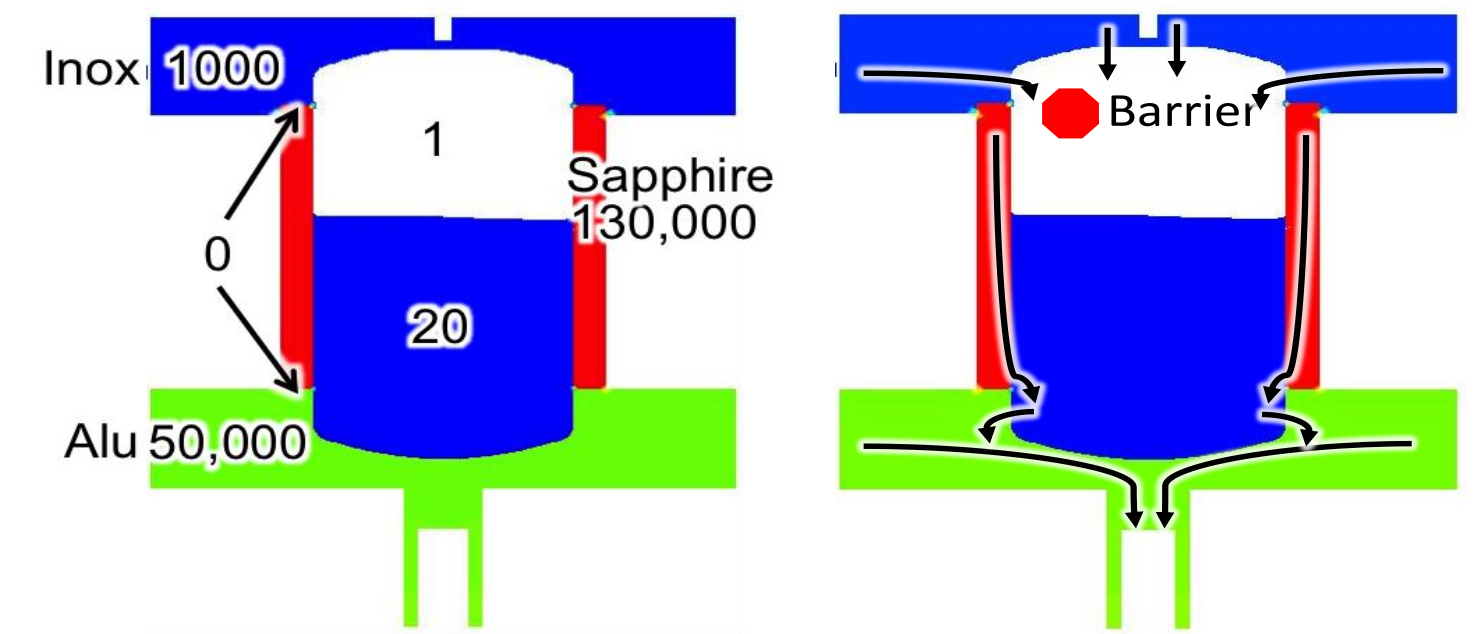

Figure 5. Relative thermal conductivity of materials (left), and flow of heat during high-g conditions (right). The sapphire cylinder and metal lids have thermal conductivities 3 orders of magnitude above the nitrogen gas; with thermal isolation at the joints, heat must diffuse through the gas, a large temperature difference develops, and a hot top lid.

Seals $^{3}$ between the sapphire shell and metal lids prevent nitrogen loss during operation, but they are also thermally insulating. Hence, heat is forced through the nitrogen vapor and liquid, and a much higher temperature gradient is required, and the top, stainless lid can be $30 \mathrm{~K}$ hotter than the sapphire and aluminum-enough to boil liquid nitrogen when it re-orients during low- $g$ conditions.

Consequently, thermal isolation of the sapphire shell and the metal lids is essential to simulating this experiment.

\section{F. What is the Experiment's Fluid/Thermal Behavior?}

The experiment's behavior is complex. During high- $g$ conditions, liquid nitrogen settles to the bottom (Figure 4), the fluid is relatively motionless, and a hot lid develops as explained in Section E. During low- $g$ conditions, acceleration reverses sign several times (Figure 3) and the liquid re-orients (i.e. from bottom-to-top) with each

\footnotetext{
${ }^{3}$ With large temperature changes and different thermal expansion coefficients, direct contact between sapphire and metal lids is hard to control, and the default design is a flexible seal between the mating surfaces and use of Belleville washers which, like springs, maintain a pre-load tension while accommodating thermal deflections. Bintley et al. [16] measured thermal conductance through sapphire-sapphire and sapphire-metal bolted joints and found good thermal isolation.
} 
acceleration sign change. Since the lid temperature is $\sim 30 \mathrm{~K}$ above the fluid saturation temperature, $T_{\text {sat }}(P)$, rapid boiling occurs when liquid nitrogen contacts the lid. Bubbles do not move away from the lid (due to buoyancy) as quickly as in 1- $g$ conditions, so larger bubbles are expected. As the hot gas bubbles move through the relatively cold liquid, there is significant vapor condensation near the liquid/vapor interface, bubbles decrease in size noticeably, and heat of condensation is transferred to the liquid. This condensation tends to balance boiling, reduces the pressure increase, and quickly moves large amounts of heat through the liquid/vapor and the apparatus.

\section{Results and Comparison with Experimental Data}

The computational results were compared with the experimental data in five ways: initial thermal profile, highspeed video, pressure evolution data, net heat transfer, and temperature probe data.

\section{G. Comparison with Initial Thermal Profile}

As noted in Section E, the boiling hot lid is established during high- $g$ intervals in the parabolic flight as the small heat flux is forced through the low thermal conductivity gas phase. This steady-state condition was simulated in a transient calculation and used as a starting point for simulation of the low-g interval. Fluid temperature sensor data is available for comparison, but wall temperature data is not. The comparison is shown in Table 1.

The agreement is very good between experimental and computational results. The disagreement is largest in the high temperature gradient (t12b) near where the top lid meets the sapphire window at a seal. A smaller discrepancy exists near the bottom wall, and it may be due to the estimated thermal boundary conditions (Section D) or the aluminum thermal conductivity.

Table 1. Comparison of experimental data and the initial thermal profile from a steady-state simulation of high-g conditions. Temperature sensor locations are shown in Figure 4. Colors indicate solid, liquid, and gas.

\begin{tabular}{|l|c|c|c|}
\hline Sensor Location & $\begin{array}{c}\text { CNES } \\
\text { Measured } \\
\text { Temperature } \\
(\mathbf{K})\end{array}$ & $\begin{array}{c}\text { Steady } \\
\text { Simulation } \\
\text { Temperature } \\
(\mathbf{K})\end{array}$ & $\begin{array}{c}\text { Percent } \\
\text { Difference } \\
(\boldsymbol{\%})\end{array}$ \\
\hline Top Lid Center & & 105.1 & \\
\hline Top Lid Edge & & 104.0 & \\
\hline Top Lid Side & & 103.3 & \\
\hline t12a & 103.77 & 104.1 & 0.3 \\
\hline t12b & 86.00 & 88.9 & 3.4 \\
\hline t12c & 77.41 & 78.1 & 0.9 \\
\hline t12d & 77.24 & 77.9 & 0.9 \\
\hline t12e & 77.21 & 77.2 & 0.0 \\
\hline t12f & 77.17 & 77.1 & -0.1 \\
\hline t12g & 77.14 & 77.0 & -0.2 \\
\hline t12h & 77.09 & 77.0 & -0.2 \\
\hline t12i & 77.02 & 76.9 & -0.2 \\
\hline t12j & 76.93 & 76.7 & -0.3 \\
\hline t12k & 76.42 & 76.3 & -0.1 \\
\hline t12l & 76.00 & 76.3 & 0.4 \\
\hline Bottom Lid Center & & 75.3 & \\
\hline
\end{tabular}

\section{H. Visual Comparison with High-Speed Video}

The high-speed video compares well with the simulation results. During the initial re-orientation, the deformation of the liquid/vapor interface (and timing) is captured well, as shown in Figure 6. The liquid/solid contact line is also captured as shown by the arrows in Figure 6.

The liquid/vapor experiences a number of re-orientations as the vertical acceleration changes sign (Figure 3), and the timing of these events is well captured. The speed of the bubbles is visually similar as acceleration changes. The simulation also captures the initiation of boiling on the top lid. As would be expected, the bubbles' size and shape are not duplicated exactly, but they are captured qualitatively (Figure 7). However, the simulation appears to have fewer bubbles than the experimental movie. The bubbles in the movie tend to be more elongated (higher aspect ratio) than those in the experiment. Experimentally, the bubbles have large, chaotic surface waves, while computationally they are relatively smooth; Figure 7 illustrates this difference. The simulation does capture the merging of bubbles. 

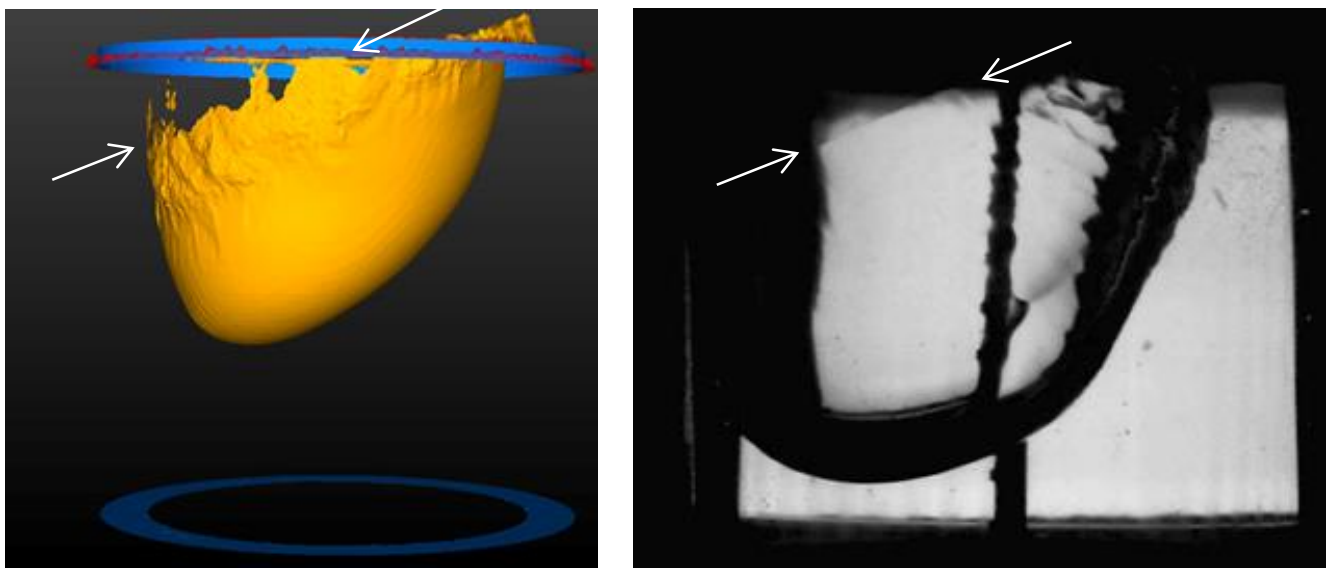

Figure 6. Comparison of computational predictions (left) and experimental data (right) showing the liquid/vapor interface as it rapidly deforms with the initial reduction in gravity. Arrows indicate the liquid/solid contact line. At left, the blue bands indicate the sapphire/lid joints.
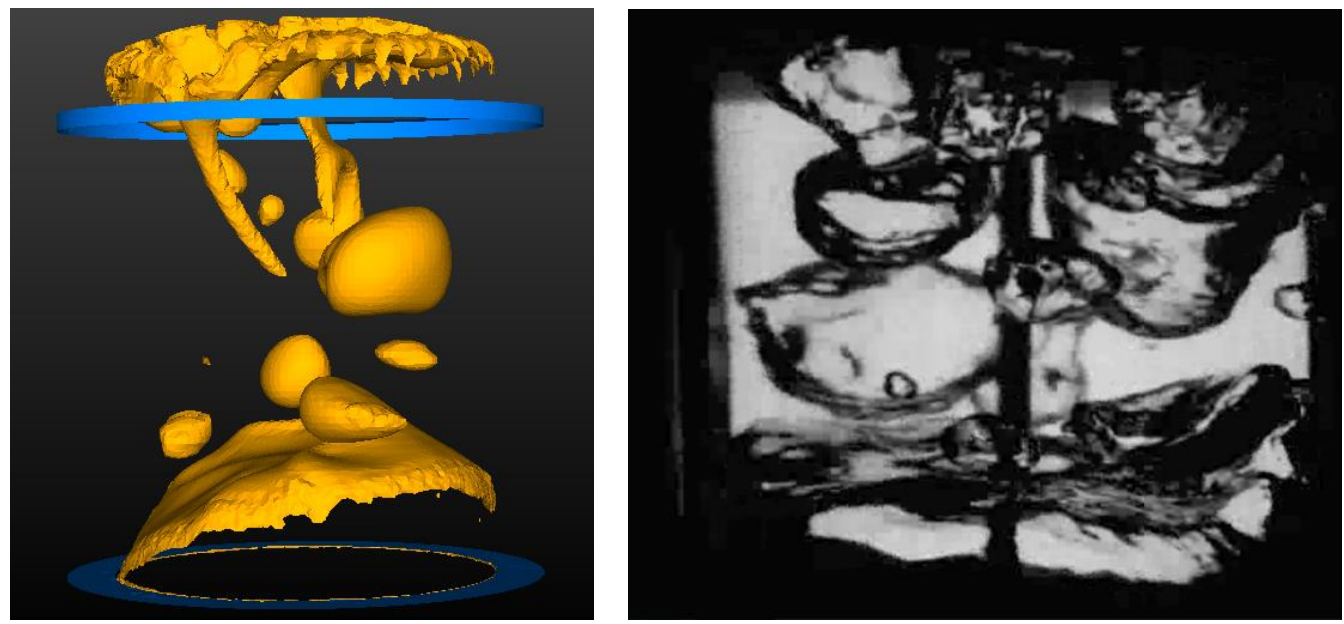

Figure 7. Comparison of computational predictions (left) and experimental data (right) showing the liquid/vapor interface during the intense boiling phase. At left, the blue bands indicate the sapphire/lid joints.

\section{Pressure Data}

Pressure is a measure of the net mass transfer due to evaporation and condensation. Figure 8 (left) shows the time evolution of mass transfer rate (evaporation, condensation, and net), and (right) shows pressure with time for two accommodation coefficients. Evaporation (boiling) precedes condensation as the liquid nitrogen contacts the top lid. After a surge of condensation and a pressure drop, evaporation and condensation reach an approximate balance with no apparent correlation with subsequent re-orientations.

The computational results for pressure, shown in Figure 8, show general agreement with the experimentally measured data. The simulation does capture the initial rapid pressure rise and reversal, but it does not capture the gentle pressure rise between 97 and 107 seconds.

\section{J. Heat Transfer Rate and Fluid Enthalpy Increase}

We verify the boiling heat transfer rate by calculating the increase in fluid enthalpy in the simulation and comparing with an estimate from the experiment. The results, shown in Figure 9, show good agreement, and indicate the accommodation coefficient value, $\sigma=5.0 \times 10^{-3}$, used in Eq. (1) is a good fit. The computational estimate evaluated the integral of Eq. (3), and the experimental estimate of enthalpy assumed the fluid was thermally stratified at the temperatures indicated by the probes before and after the low- $g$ interval.

American Institute of Aeronautics and Astronautics 

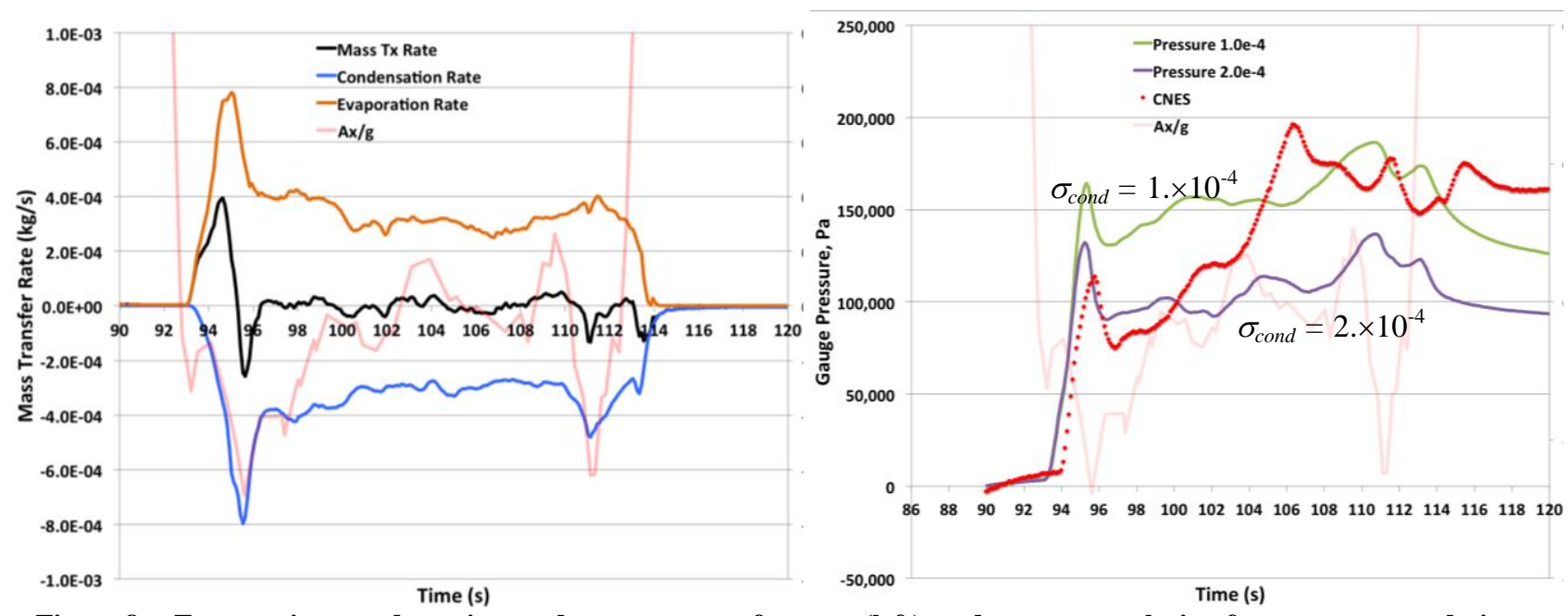

Figure 8. Evaporation, condensation, and net mass transfer rates (left), and pressure evolution for two accommodation coefficients compared with experimental measurements (right). The pale red line indicates acceleration, $a_{x} / g$.

$$
\int_{v o l} \rho_{l i q}\left(T-T_{r e f}\right) C_{v_{l i q}} V O F_{l i q} d V o l
$$

\section{K. Comparison of Temperature Probe Results}

Although experimental wall temperature data were not available, predictions from the simulation are given in Figure 10. From 114 to 120 seconds in Figure 10, the top lid is reheating. How long does the lid take to reheat during the high- $g$ interval? Combining Figure 10 data with the transient solution for initial conditions, the lid reheating time is expected to be completely reheated within 60 seconds.

The temperature evolution at two temperature probes is shown in Figure 11, and these are typical of the 12 probes. The results for probe t12a show the probe is in the hottest vapor at the top of the tank. As the hot vapor moves away and is replaced with cold liquid, the predicted quick temperature drop agrees with the experimental measurements. From 95 to 113 seconds, the liquid slowly heats, and there is general agreement between the two results. However after 115 seconds, the computation predicts re-heating of the vapor near the lid, while the experimental data do not show this. One explanation is that liquid nitrogen remains in the fill lines above probe t12a, and this liquid drains over the temperature probe after the re-orientation. The fill lines are not modeled in the

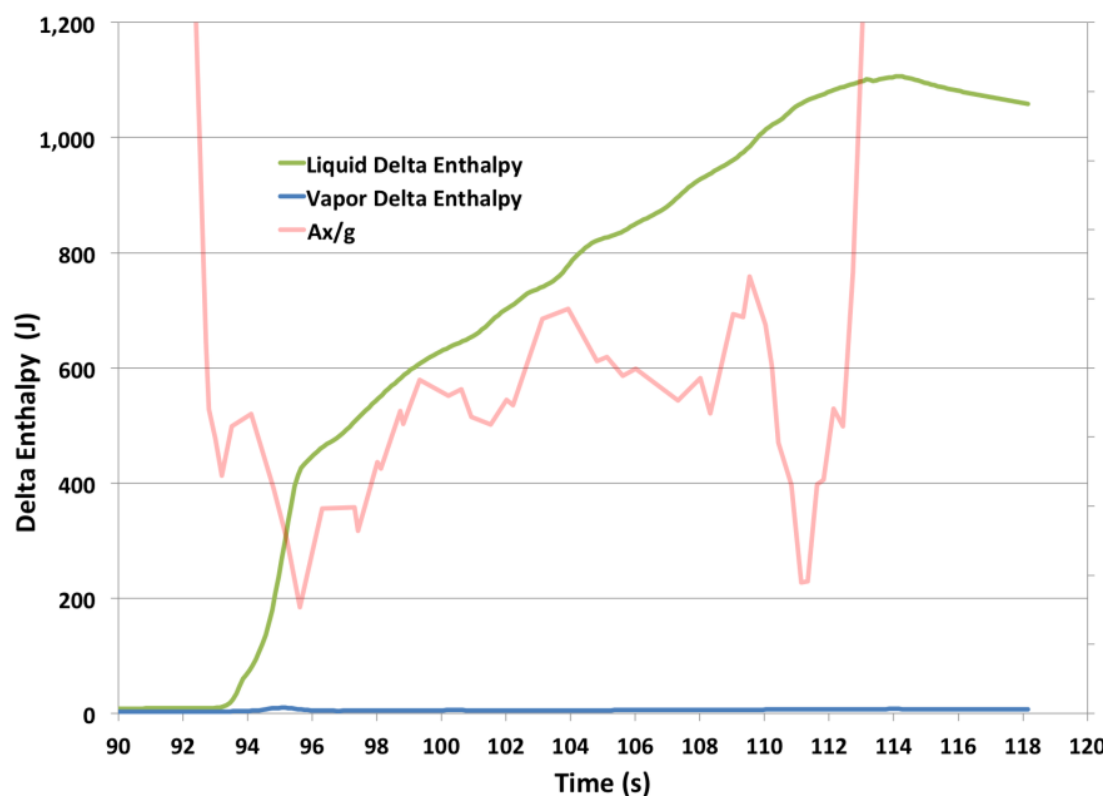

Figure 9. To check if the heat transfer rate is correct, compare the computationally measured change in fluid enthalpy with an estimate from the experiment. The pale red line indicates acceleration, $a_{x} / g$.

American Institute of Aeronautics and Astronautics 
simulation.

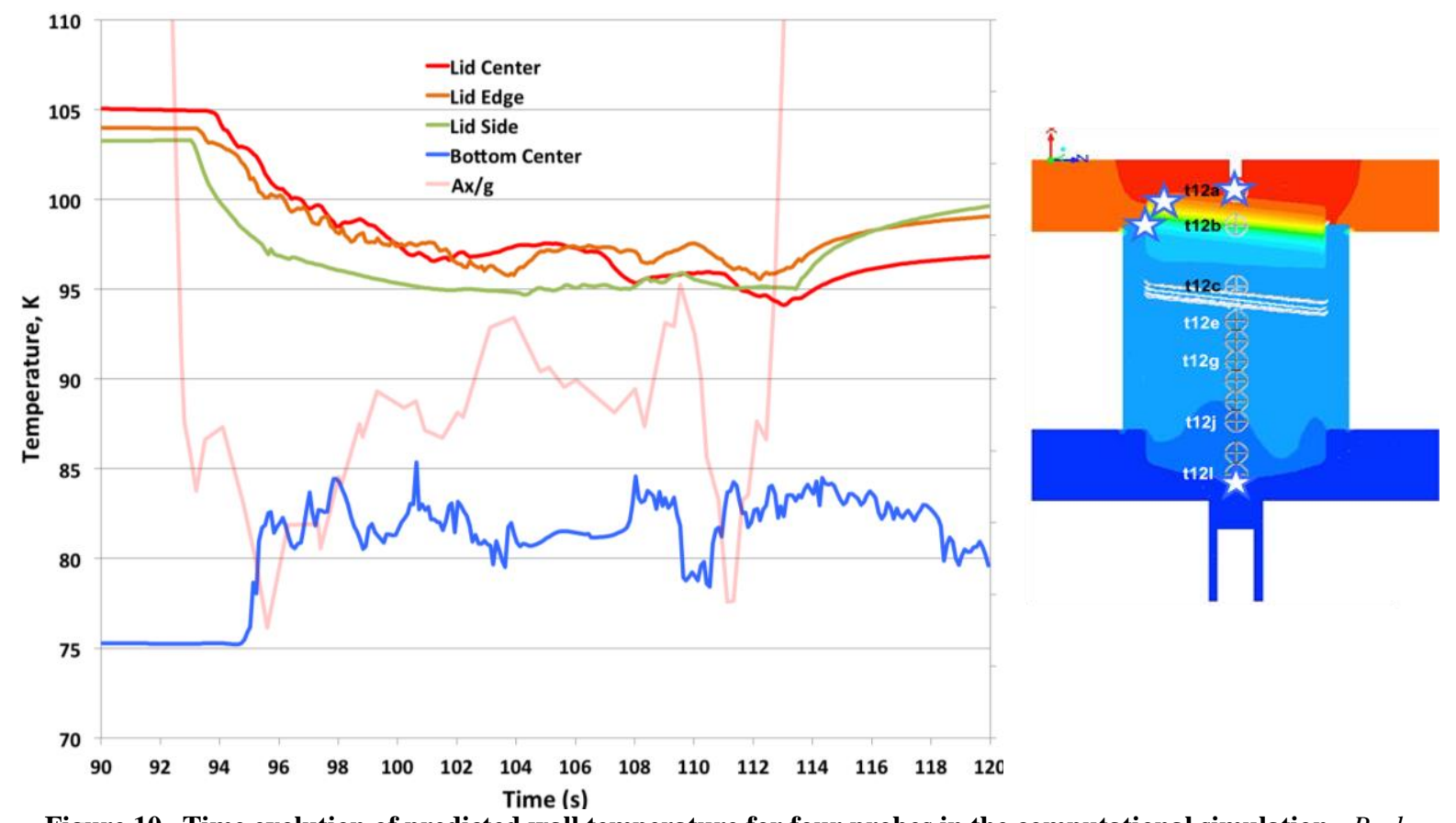

Figure 10. Time evolution of predicted wall temperature for four probes in the computational simulation. Probe locations are indicated by stars in the graphic at right. The pale red line indicates acceleration, $a_{x} / g$.

During the initial re-orientation of the fluid ( $93 \mathrm{~s})$, the hot gas at the lid moves to the bottom of the tank, passing the temperature probes mounted on the axis, and liquid nitrogen occupies the top of the tank, contacts the lid, and begins to boil. The movement of this hot gas is clear in the computational video, and in the computational temperature predictions for probe t12g, shown in Figure 11 (right) between 94 and 95 seconds. However, it is not measured by probe $\mathrm{t} 12 \mathrm{~g}$. In fact, while probes $\mathrm{t} 12 \mathrm{a}$ and $\mathrm{t} 12 \mathrm{~b}$ capture the dry-to-wet temperature jump, probes $\mathrm{t} 12 \mathrm{~d}$ to 121 do not capture the wet-to-dry temperature jump as the hot gas moves past the temperature sensors.

Typical diode temperature sensors have a time constant, $\tau$, near $0.1 \mathrm{~s}$ [16]. Consequently $95 \%$ of a temperature jump is captured in three time constants, or $0.3 \mathrm{~s}$. The computationally predicted hot vapor exposure time of the temperature probes is 0.3 to $0.5 \mathrm{~s}$, hence the sensors could detect most of the jump. However, delays in wet-to-dry
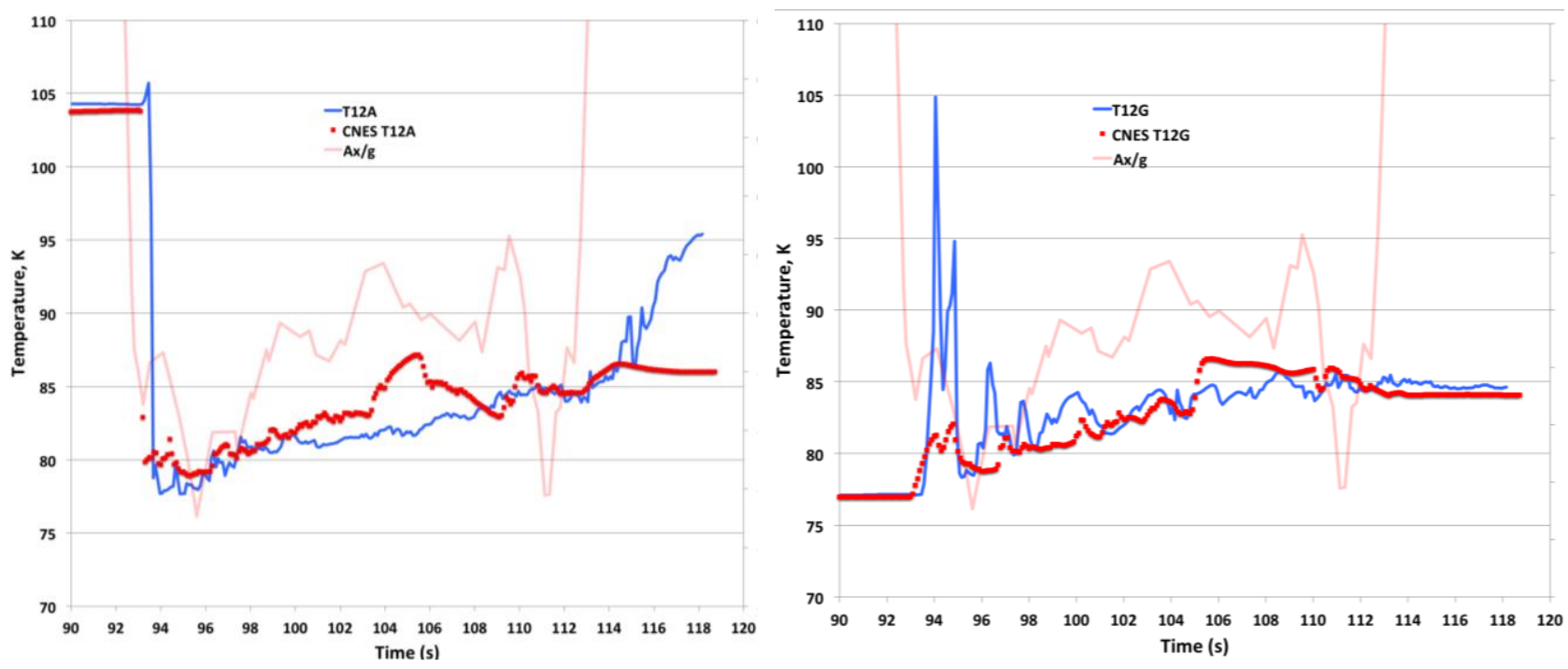

Figure 11. Predicted and measured temperatures at sensors t12a (left) and $\mathbf{t 1 2 g}$ (right). The pale red line indicates acceleration, $a_{x} / g$. 
response time have been observed experimentally and analyzed [17]. The explanation is that wet-to-dry transition of a diode sensor includes a liquid film that must vaporize before gas temperature is measured.

\section{Conclusion}

The computational and experimentally results agree. The initial temperature profile, bubble motion, and heat transfer, are in good agreement, while the pressure evolution, and shape and number of bubbles is only acceptable. Evaporation/condensation model is not predictive of rates and more theoretical analysis is needed. Turbulence damping keeps turbulence away from interface.

\section{Acknowledgments}

The authors thank Jason Hartwig, Mojib Hasan, Olga Kartuzova, and Juan Agui for helpful discussions. Steve Barsi generated the grid, Nicole Velez handled many administrative issues, and Rich Rinehart provided graphics expertise. The authors also thank Fabrice Mathey, Didier Zaepffel, and Sebastian Bianchi of ALAT for a valuable benchmarking meeting. This work was conducted under an "Implementing Arrangement Between the National Aeronautics and Space Administration of the United States of America and the Centre National D'Etudes Spatiales of France on Cooperation Related to Benchmarking Activities Regarding Computational Propellant Management Capability." The authors would like to thank Benjamin Legrand, Vincent Leudiere, and Pascal Fortunier of CNES. Without their help and discussions, this valuable cooperative activity would not have been possible. This work was supported by the NASA Space Technology Mission Directorate's Technology Demonstration Missions Program under the Evolvable Cryogenics Project.

\section{References}

[1] F. Mathey, C. Blanc-Mathieu, S. Demare, D. Zaepffel, B. Legrand and V. Leudiere, "Numerical Simulations of Two-Phase Flow Inside Cryogenic Tanks Under Microgravity Conditions: Comparison with Experiments Onboard Zero-G Parabolic Flights," in Space Propulsion Conference (Europe), Cologne, 2014.

[2] J. Lacapere, J. Tanchon, B. Legrand, Y. Prel and V. Leudiere, "Thermal Destratification Tests with Liquid Nitrogen in Parabolic Flights," in 61 st International Astronautical Congress, IAC-110.A2.3.3, 2010.

[3] B. Legrand, J. Lacapere and S. Bianchi, "New Technologies for Cryogenic Propellant Management for Next Generation Launchers," in AIAA Space, AIAA 2012-5203, Pasadena, 2012.

[4] ANSYS, ANSYS Fluent User's Guide, Release 14.0, Canonsburg, PA: ANSYS, Inc., 2011.

[5] S. W. J. Welch and J. Wilson, "A volume of fluid based method for fluid flows with phase change," Journal of Computational Physics, vol. 160, no. 2, pp. 662-682, 2000.

[6] F. R. Menter, "Two-Equation Eddy-Viscosity Turbulence Models for Engineering Applications," AIAA Journal, vol. 32 , no. 8, pp. 1598-1605, August 1994.

[7] ANSYS, ANSYS FUENT Theory Guide, Release 14.0, Canonsburg, PA: ANSYS, 2011.

[8] NIST, "Thermophysical Properties of Fluid Systems," National Institute for Standards and Technology, 2011. [Online]. Available: http://webbook.nist.gov/chemistry/fluid. [Accessed January 2015].

[9] Private Communication with CNES, 2014.

[10] NIST, "Material Measurement Laboratory: Cryogenics Technologies Group," [Online]. Available: http://cryogenics.nist.gov/MPropsMAY/materialproperties.htm. [Accessed 2015 April].

[11] Federal Aviation Administration, MMPDS-06, Metallic Materials Properties Development and Standardization, FAA, April 2011.

[12] W. C. Reynolds, Thermodynamic Properties in SI: graphs, tables and computational equations for 40 substances, Dept. of Mech Eng, Stanford University, 1979.

[13] O. Kartuzova and M. Kassemi, "Modeling Interfacial Turbulent Heat Tansfer during Ventless Pressurization of a Large Scale Cryogenic Storage Tank in Microgravity," in AIAA, 2011.

[14] K. Okuyama and Y. Iida, "Transient boiling heat transfer characteristics of nitrogen (bubble behavior and heat transfer rate at stepwise heat generation)," Int. J. Heat Mass Transfer, vol. 33, no. 10, pp. 2065-2071, 1990.

[15] A. Sakurai, M. Shiotsu and K. Hata, "Boiling heat transfer characteristics for heat inputs with various increasing rates in liquid nitrogen," Cryogenics, vol. 32, no. 5, pp. 421-429, 1992.

[16] D. Linenberger, E. Spellicy and R. Radebaugh, "Thermal Response Times of Some Cryogenic Thermometers," in 
Temperature: Its Measurement an Control in Science and Industry, American Institute of Physics, 1982, pp. $1367-1372$.

[17] E. Rame and G. A. Zimmerli, "Analysis of capillary drainage from a flat solid strip," Physics of Fluids, vol. 26, no. 062102, 2014.

[18] D. Bintley, A. L. Woodcraft and F. C. Gannaway, "Millikelvin thermal conductance measurements of compact rigid thermal isolation joints using sapphire-sapphire contacts, and of copper and beryllium-copper demountable thermal contacts," Cryogenics, vol. 47, no. 5-6, pp. 333-342, 2007. 